Supplement of

\title{
Consistency and representativeness of integrated water vapour from ground-based GPS observations and ERA-Interim reanalysis
}

\author{
Olivier Bock and Ana C. Parracho \\ Correspondence to: Olivier Bock (bock@ipgp.fr)
}

The copyright of individual parts of the supplement might differ from the CC BY 4.0 License. 
Supplement Table S1: latitude, longitude, and altitude of GPS stations, altitudes of ERA-Interim for 4 surrounding grid points sorted by distance to the GPS site, altitude difference between GPS and nearest ERA-Interim grid point, and number of days and months with valid GPS data. The nearest grid point with altitudes above or below GPS stations by more than 500m are highlighted.

\begin{tabular}{|c|c|c|c|c|c|c|c|c|c|c|}
\hline nameGPS & $\begin{array}{r}\text { latGPS } \\
\text { (deg) }\end{array}$ & $\begin{array}{r}\text { IonGPS } \\
\text { (deg) }\end{array}$ & $\begin{array}{r}\text { altGPS } \\
(\mathrm{m})\end{array}$ & $\begin{array}{l}\text { alt1 } \\
\text { (m) }\end{array}$ & $\begin{array}{l}\text { alt2 } \\
\text { (m) }\end{array}$ & $\begin{array}{l}\text { alt3 } \\
\text { (m) }\end{array}$ & $\begin{array}{l}\text { alt4 } \\
(\mathrm{m})\end{array}$ & $\begin{array}{r}\text { alt.diff. } \\
(\mathrm{m})\end{array}$ & Nday & Nmon \\
\hline albh & 48.4 & 236.5 & 51 & 255 & 273 & 218 & 251 & -204 & 5737 & 192 \\
\hline algo & 46.0 & 281.9 & 237 & 338 & 362 & 342 & 338 & -101 & 5569 & 192 \\
\hline alic & -23.7 & 133.9 & 588 & 547 & 621 & 662 & 719 & 41 & 4630 & 162 \\
\hline ankr & 39.9 & 32.8 & 939 & 1054 & 1055 & 1144 & 1072 & -115 & 3862 & 136 \\
\hline areq & -16.5 & 288.5 & 2447 & 3109 & 1803 & 4273 & 3692 & -662 & 4485 & 155 \\
\hline auck & -36.6 & 174.8 & 98 & 29 & 30 & 24 & 10 & 69 & 5174 & 174 \\
\hline azu1 & 34.1 & 242.1 & 178 & 788 & 558 & 678 & 293 & -610 & 3994 & 140 \\
\hline blyt & 33.6 & 245.3 & 119 & 341 & 424 & 209 & 256 & -222 & 5629 & 189 \\
\hline bor1 & 52.3 & 17.1 & 89 & 88 & 78 & 119 & 124 & 1 & 5560 & 191 \\
\hline bran & 34.2 & 241.7 & 280 & 678 & 293 & 788 & 558 & -398 & 5447 & 184 \\
\hline braz & -15.9 & 312.1 & 1119 & 976 & 921 & 874 & 839 & 143 & 4245 & 153 \\
\hline brmu & 32.4 & 295.3 & 23 & 0 & 0 & 0 & 0 & 23 & 5322 & 184 \\
\hline brus & 50.8 & 4.4 & 104 & 68 & 40 & 186 & 120 & 36 & 5339 & 180 \\
\hline cagl & 39.1 & 9.0 & 192 & 73 & 39 & 265 & 134 & 119 & 4954 & 172 \\
\hline cas1 & -66.3 & 110.5 & 39 & 106 & 372 & 538 & 628 & -67 & 4829 & 171 \\
\hline ccjm & 27.1 & 142.2 & 161 & 1 & 0 & -1 & 0 & 160 & 5161 & 178 \\
\hline cedu & -31.9 & 133.8 & 154 & 145 & 26 & 175 & 106 & 9 & 3775 & 133 \\
\hline cfag & -31.6 & 291.8 & 680 & 995 & 675 & 859 & 522 & -315 & 4713 & 162 \\
\hline chat & -44.0 & 183.4 & 48 & 1 & 1 & 1 & 1 & 47 & 5170 & 173 \\
\hline chil & 34.3 & 242.0 & 1601 & 788 & 678 & 558 & 293 & 813 & 5158 & 173 \\
\hline chur & 58.8 & 265.9 & 29 & 19 & 50 & -1 & 8 & 10 & 5422 & 186 \\
\hline cit1 & 34.1 & 241.9 & 249 & 678 & 788 & 293 & 558 & -429 & 4902 & 166 \\
\hline clar & 34.1 & 242.3 & 407 & 558 & 788 & 794 & 885 & -151 & 5242 & 177 \\
\hline cmp9 & 34.4 & 241.6 & 1172 & 678 & 788 & 293 & 558 & 494 & 5104 & 175 \\
\hline coco & -12.2 & 96.8 & 5 & 0 & 0 & 0 & 0 & 5 & 4342 & 154 \\
\hline coso & 36.0 & 242.2 & 1485 & 1322 & 1327 & 1014 & 967 & 163 & 4787 & 161 \\
\hline $\operatorname{crfp}$ & 34.0 & 242.9 & 721 & 794 & 885 & 558 & 788 & -73 & 5597 & 186 \\
\hline cro1 & 17.8 & 295.4 & 13 & -1 & -2 & 15 & 5 & 14 & 4212 & 149 \\
\hline $\operatorname{csn} 1$ & 34.3 & 241.5 & 296 & 678 & 293 & 648 & 118 & -382 & 4574 & 156 \\
\hline darw & -12.8 & 131.1 & 75 & 99 & 64 & 154 & 108 & -24 & 3669 & 128 \\
\hline dav1 & -68.6 & 78.0 & 27 & 56 & 3 & 686 & 323 & -29 & 4168 & 147 \\
\hline dgar & -7.3 & 72.4 & 10 & 0 & 0 & 0 & 0 & 10 & 3342 & 113 \\
\hline dhlg & 33.4 & 244.2 & -49 & 447 & 313 & 677 & 508 & -496 & 4890 & 169 \\
\hline drao & 49.3 & 240.4 & 558 & 1314 & 1277 & 1292 & 1132 & -719 & 5751 & 192 \\
\hline dubo & 50.3 & 264.1 & 275 & 278 & 321 & 273 & 324 & -3 & 4937 & 165 \\
\hline ebre & 40.8 & 0.5 & 58 & 111 & 334 & 475 & 467 & -53 & 4999 & 174 \\
\hline fair & 65.0 & 212.5 & 308 & 391 & 468 & 487 & 548 & -83 & 4972 & 169 \\
\hline fale & -13.8 & 188.0 & 14 & 36 & 20 & 40 & 22 & -22 & 3694 & 132 \\
\hline flin & 54.7 & 258.0 & 343 & 307 & 295 & 302 & 284 & 36 & 4777 & 167 \\
\hline gode & 39.0 & 283.2 & 48 & 46 & 111 & 126 & 193 & 2 & 4826 & 169 \\
\hline gold & 35.4 & 243.1 & 1018 & 877 & 847 & 1025 & 961 & 141 & 4091 & 138 \\
\hline gope & 49.9 & 14.8 & 547 & 349 & 482 & 371 & 494 & 198 & 4781 & 165 \\
\hline gras & 43.8 & 6.9 & 1269 & 665 & 164 & 1137 & 785 & 604 & 4651 & 161 \\
\hline graz & 47.1 & 15.5 & 491 & 601 & 883 & 436 & 673 & -110 & 5171 & 184 \\
\hline guam & 13.6 & 144.9 & 147 & 3 & 1 & 2 & 0 & 144 & 4321 & 144 \\
\hline hers & 50.9 & 0.3 & 32 & 35 & 29 & 7 & 33 & -3 & 4804 & 163 \\
\hline hob2 & -42.8 & 147.4 & 45 & 249 & 365 & 53 & 74 & -204 & 4794 & 167 \\
\hline holb & 50.6 & 231.9 & 576 & 55 & 61 & 145 & 271 & 521 & 5124 & 172 \\
\hline holp & 33.9 & 241.8 & 29 & 293 & 558 & 678 & 788 & -264 & 5608 & 188 \\
\hline hrao & -25.9 & 27.7 & 1389 & 1472 & 1245 & 1442 & 1239 & -83 & 4447 & 150 \\
\hline iisc & 13.0 & 77.6 & 930 & 787 & 717 & 767 & 725 & 143 & 3497 & 125 \\
\hline irkt & 52.2 & 104.3 & 541 & 627 & 958 & 655 & 849 & -86 & 4949 & 179 \\
\hline joze & 52.1 & 21.0 & 110 & 158 & 120 & 161 & 136 & -48 & 5568 & 191 \\
\hline jplm & 34.2 & 241.8 & 458 & 678 & 788 & 293 & 558 & -220 & 5155 & 174 \\
\hline karr & -21.0 & 117.1 & 117 & 122 & 143 & -1 & 2 & -5 & 4785 & 170 \\
\hline kely & 67.0 & 309.1 & 198 & 673 & 806 & 447 & 618 & -475 & 2175 & 94 \\
\hline kerg & -49.4 & 70.3 & 33 & 34 & 84 & 12 & 27 & -1 & 4837 & 164 \\
\hline kiru & 67.9 & 21.0 & 362 & 429 & 557 & 523 & 634 & -67 & 5120 & 183 \\
\hline kit3 & 39.1 & 66.9 & 659 & 1178 & 1928 & 863 & 1250 & -519 & 4424 & 166 \\
\hline
\end{tabular}




\begin{tabular}{|c|c|c|c|c|c|c|c|c|c|c|}
\hline nameGPS & $\begin{array}{r}\text { latGPS } \\
\text { (deg) } \\
\end{array}$ & $\begin{array}{r}\text { IonGPS } \\
\text { (deg) }\end{array}$ & $\begin{array}{r}\text { altGPS } \\
(\mathrm{m})\end{array}$ & $\begin{array}{l}\text { alt1 } \\
(\mathrm{m})\end{array}$ & $\begin{array}{l}\text { alt2 } \\
(\mathrm{m})\end{array}$ & $\begin{array}{l}\text { alt3 } \\
(\mathrm{m})\end{array}$ & $\begin{array}{l}\text { alt4 } \\
(\mathrm{m}) \\
\end{array}$ & $\begin{array}{r}\text { alt.diff. } \\
(\mathrm{m})\end{array}$ & Nday & Nmon \\
\hline kokb & 22.1 & 200.3 & 1153 & 22 & 24 & 11 & 12 & 1131 & 4580 & 156 \\
\hline kosg & 52.2 & 5.8 & 53 & 9 & 27 & -2 & 10 & 44 & 5181 & 179 \\
\hline kour & 5.3 & 307.2 & 8 & 9 & 43 & -1 & 4 & -1 & 4467 & 163 \\
\hline lama & 53.9 & 20.7 & 158 & 110 & 93 & 132 & 123 & 48 & 5197 & 176 \\
\hline lbch & 33.8 & 241.8 & 8 & 293 & 558 & 678 & 788 & -285 & 4553 & 156 \\
\hline leep & 34.1 & 241.7 & 520 & 678 & 293 & 788 & 558 & -158 & 5538 & 186 \\
\hline long & 34.1 & 242.0 & 108 & 558 & 788 & 293 & 678 & -450 & 5182 & 176 \\
\hline Ipgs & -34.9 & 302.1 & 14 & 9 & 22 & 14 & 19 & 5 & 4125 & 150 \\
\hline mac1 & -54.5 & 158.9 & 14 & 1 & 0 & 0 & 1 & 14 & 4647 & 167 \\
\hline madr & 40.4 & 355.8 & 777 & 854 & 851 & 684 & 715 & -77 & 4477 & 156 \\
\hline mas1 & 27.8 & 344.4 & 155 & 53 & 13 & 65 & 7 & 102 & 5087 & 175 \\
\hline mate & 40.6 & 16.7 & 490 & 303 & 85 & 112 & 17 & 187 & 5156 & 176 \\
\hline maw1 & -67.6 & 62.9 & 30 & 497 & 536 & 1182 & 1238 & -467 & 4581 & 169 \\
\hline $\mathrm{mcm} 4$ & -77.8 & 166.7 & 151 & 187 & 112 & 117 & 48 & -36 & 4196 & 146 \\
\hline mdo1 & 30.7 & 256.0 & 2027 & 1319 & 1176 & 1298 & 1182 & 708 & 5002 & 171 \\
\hline medi & 44.5 & 11.6 & 10 & 270 & 253 & 112 & 176 & -260 & 5015 & 175 \\
\hline mets & 60.2 & 24.4 & 76 & 31 & 31 & 82 & 88 & 46 & 4864 & 168 \\
\hline mkea & 19.8 & 204.5 & 3730 & 563 & 176 & 496 & 221 & 3167 & 4440 & 149 \\
\hline monp & 32.9 & 243.6 & 1875 & 508 & 539 & 561 & 389 & 1367 & 5300 & 180 \\
\hline nano & 49.3 & 235.9 & 24 & 405 & 385 & 255 & 226 & -381 & 5438 & 183 \\
\hline nlib & 41.8 & 268.4 & 240 & 257 & 222 & 275 & 248 & -17 & 4607 & 160 \\
\hline nrc1 & 45.5 & 284.4 & 116 & 198 & 144 & 206 & 178 & -82 & 5280 & 176 \\
\hline onsa & 57.4 & 11.9 & 9 & 70 & 38 & 9 & 13 & -61 & 4988 & 170 \\
\hline penc & 47.8 & 19.3 & 248 & 276 & 247 & 126 & 151 & -28 & 4969 & 173 \\
\hline pert & -31.8 & 115.9 & 45 & 212 & 62 & 205 & 48 & -167 & 5068 & 177 \\
\hline pie1 & 34.3 & 251.9 & 2370 & 2193 & 2184 & 2161 & 2178 & 177 & 4857 & 164 \\
\hline pin1 & 33.6 & 243.5 & 1289 & 677 & 794 & 508 & 539 & 612 & 5562 & 191 \\
\hline pol2 & 42.7 & 74.7 & 1755 & 1865 & 1793 & 2605 & 2502 & -110 & 4495 & 152 \\
\hline pots & 52.4 & 13.1 & 104 & 55 & 51 & 112 & 110 & 50 & 5374 & 186 \\
\hline quin & 40.0 & 239.1 & 1129 & 1369 & 820 & 1554 & 1197 & -240 & 3973 & 137 \\
\hline reyk & 64.1 & 338.0 & 27 & 245 & 41 & 71 & 25 & -218 & 4787 & 169 \\
\hline rock & 34.2 & 241.3 & 588 & 678 & 293 & 648 & 118 & -90 & 5287 & 180 \\
\hline sant & -33.1 & 289.3 & 696 & 2153 & 671 & 1980 & 566 & -1457 & 4394 & 152 \\
\hline shao & 31.1 & 121.2 & 12 & 12 & 0 & 20 & 3 & 0 & 3379 & 118 \\
\hline sio3 & 32.9 & 242.8 & 70 & 539 & 149 & 389 & 15 & -469 & 5549 & 189 \\
\hline sni1 & 33.2 & 240.5 & 277 & -20 & -14 & 118 & 44 & 297 & 4187 & 139 \\
\hline spk1 & 34.1 & 241.4 & 476 & 293 & 678 & 118 & 648 & 183 & 4838 & 165 \\
\hline stjo & 47.6 & 307.3 & 143 & 17 & 17 & 52 & 63 & 126 & 5536 & 190 \\
\hline svtl & 60.5 & 29.8 & 61 & 30 & 33 & 34 & 31 & 31 & 4932 & 169 \\
\hline syog & -69.0 & 39.6 & 28 & 429 & 157 & 698 & 412 & -401 & 4875 & 173 \\
\hline tabl & 34.4 & 242.3 & 2261 & 788 & 885 & 558 & 794 & 1473 & 5206 & 174 \\
\hline tidb & -35.4 & 149.0 & 646 & 682 & 655 & 774 & 863 & -36 & 4647 & 158 \\
\hline tow2 & -19.3 & 147.1 & 30 & 177 & 37 & 26 & -4 & -147 & 5199 & 182 \\
\hline trak & 33.6 & 242.2 & 151 & 558 & 293 & 149 & -8 & -407 & 5461 & 189 \\
\hline tskb & 36.1 & 140.1 & 28 & 109 & 362 & 344 & 585 & -81 & 5143 & 174 \\
\hline uclp & 34.1 & 241.6 & 147 & 293 & 678 & 558 & 788 & -146 & 5031 & 168 \\
\hline uclu & 48.9 & 234.5 & 29 & 119 & 79 & 351 & 343 & -90 & 5510 & 184 \\
\hline usc1 & 34.0 & 241.7 & 57 & 293 & 678 & 558 & 788 & -236 & 5079 & 170 \\
\hline usud & 36.1 & 138.4 & 1466 & 990 & 765 & 774 & 739 & 476 & 4868 & 164 \\
\hline vill & 40.4 & 356.0 & 595 & 851 & 854 & 715 & 684 & -256 & 5126 & 181 \\
\hline vndp & 34.6 & 239.4 & 25 & 88 & 398 & 259 & 369 & -63 & 5186 & 178 \\
\hline wes2 & 42.6 & 288.5 & 114 & 113 & 250 & 60 & 149 & 1 & 4569 & 165 \\
\hline whc1 & 34.0 & 242.0 & 129 & 558 & 293 & 788 & 678 & -429 & 5132 & 172 \\
\hline whit & 60.8 & 224.8 & 1420 & 1108 & 1132 & 1060 & 1045 & 313 & 4970 & 168 \\
\hline will & 52.2 & 237.8 & 1110 & 1046 & 1150 & 1175 & 1128 & 64 & 5550 & 184 \\
\hline wlsn & 34.2 & 241.9 & 1739 & 788 & 678 & 558 & 293 & 951 & 4787 & 160 \\
\hline wslr & 50.1 & 237.1 & 926 & 1344 & 1347 & 593 & 832 & -418 & 5097 & 170 \\
\hline wtzr & 49.1 & 12.9 & 619 & 507 & 477 & 507 & 533 & 112 & 5094 & 174 \\
\hline wuhn & 30.5 & 114.4 & 40 & 57 & 102 & 95 & 140 & -17 & 4955 & 173 \\
\hline yell & 62.5 & 245.5 & 207 & 184 & 217 & 243 & 273 & 23 & 5678 & 192 \\
\hline zimm & 46.9 & 7.5 & 907 & 777 & 1378 & 615 & 1100 & 130 & 5636 & 192 \\
\hline
\end{tabular}


Supplement Table S2: mean GPS IWV, standard deviation of GPS IWV, mean GPS formal error, absolute and relative mean deviation of IWV from 4 grid points, absolute and relative mean difference of IWV (ERA-Interim - GPS), absolute and relative standard deviation of difference of IWV (ERA-Interim - GPS), minimum absolute and relative standard deviation of difference of IWV (ERA-Interim - GPS) from 4 grid points. Differences exceeding the acceptance limit and outlying stations are highlighted in red.

\begin{tabular}{|c|c|c|c|c|c|c|c|c|c|c|c|}
\hline \multirow[t]{2}{*}{ nameGPS } & meanGPS & stdGPS & errGPS & meanR & relmeanR & meanD & relmeanD & stdD & relstdD & \multicolumn{2}{|c|}{$\min 4 s t d D \min 4$ relstdD } \\
\hline & $(\mathrm{kg} / \mathrm{m} 2)$ & $(\mathrm{kg} / \mathrm{m} 2)$ & $(\mathrm{kg} / \mathrm{m} 2)$ & $(\mathrm{kg} / \mathrm{m} 2)$ & (\%) & $(\mathrm{kg} / \mathrm{m} 2)$ & (\%) & $(\mathrm{kg} / \mathrm{m} 2)$ & (\%) & $(\mathrm{kg} / \mathrm{m} 2)$ & (\%) \\
\hline albh & 15.25 & 5.76 & 0.28 & 1.78 & 11.1 & 0.88 & 5.80 & 1.28 & 8.40 & 1.32 & 8.70 \\
\hline algo & 14.11 & 9.45 & 0.28 & 1.71 & 11.5 & 0.69 & 4.90 & 1.00 & 7.10 & 1.05 & 7.50 \\
\hline alic & 18.04 & 10.70 & 0.29 & 2.02 & 11.4 & -0.28 & -1.60 & 1.68 & 9.30 & 1.83 & 10.20 \\
\hline ankr & 12.89 & 5.60 & 0.28 & 1.52 & 11.2 & 0.63 & 4.90 & 1.06 & 8.20 & 1.09 & 8.50 \\
\hline areq & 11.62 & 6.66 & 0.35 & 6.41 & 55.1 & 0.01 & 0.10 & 2.43 & 20.90 & 2.55 & 22.00 \\
\hline auck & 18.40 & 6.98 & 0.27 & 2.17 & 11.5 & 0.40 & 2.20 & 0.87 & 4.70 & 0.91 & 4.90 \\
\hline azu1 & 16.01 & 7.43 & 0.37 & 2.23 & 14.6 & -0.72 & -4.50 & 2.02 & 12.60 & 2.07 & 12.90 \\
\hline blyt & 15.56 & 10.19 & 0.27 & 2.23 & 14.3 & 0.06 & 0.40 & 2.30 & 14.80 & 2.33 & 15.00 \\
\hline bor1 & 15.11 & 7.69 & 0.29 & 1.53 & 9.6 & 0.77 & 5.10 & 0.81 & 5.30 & 0.83 & 5.50 \\
\hline bran & 15.24 & 6.84 & 0.38 & 2.26 & 15.3 & -0.46 & -3.00 & 1.80 & 11.80 & 1.89 & 12.40 \\
\hline braz & 27.08 & 9.58 & 0.31 & 2.10 & 7.9 & -0.55 & -2.00 & 1.87 & 6.90 & 1.88 & 6.90 \\
\hline brmu & 29.70 & 11.33 & 0.35 & 2.84 & 9.5 & 0.18 & 0.60 & 1.21 & 4.10 & 1.33 & 4.50 \\
\hline brus & 15.78 & 6.87 & 0.29 & 1.72 & 10.3 & 0.86 & 5.50 & 0.77 & 4.90 & 0.81 & 5.10 \\
\hline cagl & 18.03 & 6.48 & 0.29 & 2.26 & 12.5 & 0.15 & 0.80 & 1.10 & 6.10 & 1.15 & 6.40 \\
\hline cas1 & 4.21 & 2.08 & 0.25 & 0.69 & 16.4 & -0.03 & -0.70 & 0.65 & 15.40 & 0.68 & 16.20 \\
\hline ccjm & 34.75 & 13.04 & 0.32 & 3.30 & 9.6 & -0.39 & -1.10 & 1.80 & 5.20 & 1.90 & 5.50 \\
\hline cedu & 16.08 & 7.01 & 0.27 & 1.98 & 12.3 & 0.01 & 0.10 & 1.17 & 7.30 & 1.32 & 8.20 \\
\hline cfag & 16.57 & 7.83 & 0.30 & 3.49 & 15.6 & 5.76 & 34.80 & 3.67 & 22.20 & 3.50 & 21.10 \\
\hline chat & 15.41 & 5.88 & 0.29 & 1.68 & 10.7 & 0.23 & 1.50 & 0.77 & 5.00 & 0.85 & 5.50 \\
\hline chil & 7.74 & 4.63 & 0.32 & 1.60 & 21.0 & -0.09 & -1.20 & 0.94 & 12.20 & 0.99 & 12.80 \\
\hline chur & 9.24 & 7.35 & 0.28 & 1.18 & 12.0 & 0.58 & 6.20 & 0.67 & 7.30 & 0.77 & 8.30 \\
\hline cit1 & 15.02 & 7.01 & 0.28 & 2.26 & 15.1 & -0.05 & -0.30 & 1.84 & 12.20 & 1.91 & 12.70 \\
\hline clar & 14.52 & 6.89 & 0.28 & 2.19 & 15.6 & -0.54 & -3.70 & 1.83 & 12.60 & 1.90 & 13.10 \\
\hline cmp9 & 9.88 & 5.15 & 0.30 & 1.94 & 20.3 & -0.31 & -3.10 & 1.15 & 11.60 & 1.20 & 12.10 \\
\hline coco & 42.58 & 9.84 & 0.28 & 3.03 & 7.2 & -0.43 & -1.00 & 1.53 & 3.60 & 1.62 & 3.80 \\
\hline coso & 8.00 & 4.61 & 0.27 & 1.74 & 18.6 & 1.37 & 17.20 & 1.33 & 16.60 & 1.34 & 16.70 \\
\hline crfp & 12.83 & 6.65 & 0.27 & 2.03 & 16.6 & -0.59 & -4.60 & 1.72 & 13.40 & 1.82 & 14.10 \\
\hline cro1 & 37.92 & 8.44 & 0.45 & 2.71 & 7.0 & 0.69 & 1.80 & 1.66 & 4.40 & 1.74 & 4.60 \\
\hline $\operatorname{csn} 1$ & 14.19 & 6.66 & 0.27 & 2.42 & 16.8 & 0.21 & 1.50 & 1.73 & 12.20 & 1.82 & 12.80 \\
\hline darw & 38.22 & 14.37 & 0.32 & 3.52 & 9.3 & -0.25 & -0.70 & 1.71 & 4.50 & 1.82 & 4.80 \\
\hline dav1 & 3.74 & 1.86 & 0.25 & 0.51 & 14.7 & -0.26 & -7.00 & 0.45 & 12.10 & 0.47 & 12.60 \\
\hline dgar & 48.29 & 8.10 & 0.29 & 2.87 & 6.1 & -0.83 & -1.70 & 1.88 & 3.90 & 2.00 & 4.10 \\
\hline dhlg & 16.23 & 10.23 & 0.26 & 2.63 & 15.5 & 0.69 & 4.30 & 2.70 & 16.60 & 2.64 & 16.30 \\
\hline drao & 12.28 & 5.25 & 0.25 & 1.32 & 10.1 & 0.72 & 5.80 & 1.17 & 9.50 & 1.23 & 10.00 \\
\hline dubo & 12.61 & 8.97 & 0.30 & 1.48 & 10.9 & 0.95 & 7.50 & 1.07 & 8.40 & 1.08 & 8.50 \\
\hline ebre & 19.36 & 8.64 & 0.28 & 2.78 & 13.8 & 0.71 & 3.70 & 1.73 & 9.00 & 1.90 & 9.80 \\
\hline fair & 8.84 & 6.43 & 0.31 & 0.93 & 9.6 & 0.85 & 9.60 & 0.94 & 10.60 & 0.99 & 11.10 \\
\hline fale & 47.62 & 8.94 & 0.26 & 3.01 & 6.3 & -0.02 & 0.00 & 1.86 & 3.90 & 2.10 & 4.40 \\
\hline flin & 11.22 & 7.77 & 0.27 & 1.28 & 10.8 & 0.69 & 6.10 & 0.92 & 8.20 & 0.92 & 8.20 \\
\hline gode & 20.49 & 12.49 & 0.30 & 2.45 & 11.5 & 0.88 & 4.30 & 1.13 & 5.50 & 1.20 & 5.90 \\
\hline gold & 9.83 & 5.56 & 0.29 & 1.61 & 15.1 & 0.82 & 8.40 & 1.21 & 12.30 & 1.20 & 12.20 \\
\hline gope & 13.82 & 6.79 & 0.34 & 1.54 & 11.0 & 0.24 & 1.80 & 0.81 & 5.90 & 0.90 & 6.50 \\
\hline gras & 10.19 & 5.35 & 0.28 & 2.06 & 19.3 & 0.48 & 4.70 & 1.10 & 10.80 & 1.25 & 12.30 \\
\hline graz & 14.87 & 7.66 & 0.27 & 1.90 & 11.9 & 1.09 & 7.30 & 1.04 & 7.00 & 1.11 & 7.50 \\
\hline guam & 44.46 & 9.76 & 0.36 & 2.93 & 6.7 & -0.63 & -1.40 & 1.59 & 3.60 & 1.61 & 3.60 \\
\hline hers & 16.20 & 6.58 & 0.26 & 1.91 & 11.3 & 0.81 & 5.00 & 0.80 & 4.90 & 0.86 & 5.30 \\
\hline hob2 & 14.94 & 5.13 & 0.26 & 1.77 & 11.1 & 0.98 & 6.60 & 0.84 & 5.60 & 0.84 & 5.60 \\
\hline holb & 11.99 & 5.21 & 0.30 & 1.56 & 12.8 & 0.16 & 1.30 & 0.76 & 6.40 & 0.86 & 7.20 \\
\hline holp & 16.77 & 7.15 & 0.31 & 2.40 & 14.4 & -0.14 & -0.90 & 1.76 & 10.50 & 1.79 & 10.70 \\
\hline hrao & 16.88 & 8.81 & 0.33 & 1.99 & 11.6 & 0.31 & 1.80 & 1.25 & 7.40 & 1.40 & 8.30 \\
\hline iisc & 31.44 & 11.35 & 0.32 & 2.52 & 8.0 & 0.14 & 0.50 & 2.30 & 7.30 & 2.37 & 7.50 \\
\hline irkt & 10.29 & 8.89 & 0.33 & 1.28 & 11.3 & 0.99 & 9.60 & 1.25 & 12.20 & 1.24 & 12.00 \\
\hline joze & 14.93 & 7.85 & 0.33 & 1.50 & 9.5 & 0.90 & 6.00 & 0.83 & 5.60 & 0.93 & 6.20 \\
\hline jplm & 14.05 & 6.62 & 0.39 & 2.20 & 16.3 & -0.52 & -3.70 & 1.70 & 12.10 & 1.82 & 13.00 \\
\hline karr & 24.17 & 12.86 & 0.30 & 2.98 & 12.3 & -0.01 & 0.00 & 1.70 & 7.00 & 1.70 & 7.00 \\
\hline kely & 7.70 & 4.68 & 0.30 & 0.82 & 9.9 & 0.64 & 8.40 & 0.86 & 11.20 & 0.88 & 11.50 \\
\hline kerg & 10.23 & 4.40 & 0.34 & 1.38 & 12.6 & 0.73 & 7.10 & 0.95 & 9.30 & 0.96 & 9.40 \\
\hline kiru & 9.25 & 5.79 & 0.31 & 0.87 & 8.6 & 0.96 & 10.30 & 0.84 & 9.10 & 0.87 & 9.40 \\
\hline kit3 & 13.68 & 5.89 & 0.43 & 4.12 & 25.2 & 2.70 & 19.70 & 3.59 & 26.20 & 3.16 & 23.10 \\
\hline
\end{tabular}




\begin{tabular}{|c|c|c|c|c|c|c|c|c|c|c|c|}
\hline \multirow[t]{2}{*}{ nameGPS } & \multirow{2}{*}{$\begin{array}{r}\text { meanGPS } \\
(\mathrm{kg} / \mathrm{m} 2)\end{array}$} & \multirow{2}{*}{$\begin{array}{l}\text { stdGPS } \\
(\mathrm{kg} / \mathrm{m} 2)\end{array}$} & \multirow{2}{*}{$\begin{array}{r}\text { errGPS } \\
(\mathrm{kg} / \mathrm{m} 2)\end{array}$} & \multirow{2}{*}{$\begin{array}{r}\text { meanR } \\
(\mathrm{kg} / \mathrm{m} 2)\end{array}$} & \multirow{2}{*}{$\begin{array}{r}\text { relmeanR } \\
(\%)\end{array}$} & \multirow{2}{*}{$\begin{array}{r}\text { meanD } \\
(\mathrm{kg} / \mathrm{m} 2)\end{array}$} & \multirow{2}{*}{$\begin{array}{r}\text { relmeanD } \\
(\%)\end{array}$} & \multirow{2}{*}{$\begin{array}{r}\text { stdD } \\
(\mathrm{kg} / \mathrm{m} 2)\end{array}$} & \multirow{2}{*}{$\begin{array}{r}\text { relstdD } \\
(\%)\end{array}$} & \multicolumn{2}{|c|}{$\min 4 s t d D$ min4relstdD } \\
\hline & & & & & & & & & & $(\mathrm{kg} / \mathrm{m} 2)$ & (\%) \\
\hline kokb & 15.15 & 5.41 & 0.33 & 1.84 & 12.2 & -0.09 & -0.60 & 1.23 & 8.10 & 1.35 & 8.90 \\
\hline kosg & 16.10 & 6.91 & 0.25 & 1.72 & 10.4 & 0.51 & 3.10 & 0.70 & 4.40 & 0.82 & 5.10 \\
\hline kour & 48.32 & 6.58 & 0.34 & 3.07 & 6.4 & -0.57 & -1.20 & 1.76 & 3.60 & 1.81 & 3.70 \\
\hline lama & 14.22 & 7.44 & 0.26 & 1.48 & 10.0 & 0.64 & 4.50 & 0.87 & 6.10 & 0.88 & 6.20 \\
\hline Ibch & 16.77 & 7.27 & 0.36 & 2.43 & 14.4 & 0.05 & 0.30 & 1.72 & 10.20 & 1.74 & 10.40 \\
\hline leep & 12.67 & 6.19 & 0.28 & 2.19 & 16.8 & 0.34 & 2.70 & 1.53 & 12.10 & 1.59 & 12.50 \\
\hline long & 16.65 & 7.35 & 0.43 & 2.31 & 14.5 & -0.73 & -4.40 & 2.24 & 13.40 & 2.32 & 14.00 \\
\hline Ipgs & 20.36 & 9.27 & 0.46 & 2.79 & 13.3 & 0.65 & 3.20 & 1.54 & 7.60 & 1.67 & 8.20 \\
\hline mac1 & 10.95 & 3.93 & 0.27 & 1.24 & 11.0 & 0.32 & 2.90 & 0.58 & 5.30 & 0.62 & 5.70 \\
\hline madr & 13.80 & 5.59 & 0.31 & 1.65 & 11.5 & 0.55 & 4.00 & 1.13 & 8.20 & 1.16 & 8.40 \\
\hline mas1 & 18.18 & 6.29 & 0.37 & 1.95 & 10.4 & 0.66 & 3.60 & 1.36 & 7.50 & 1.36 & 7.50 \\
\hline mate & 15.80 & 6.38 & 0.33 & 1.99 & 12.2 & 0.47 & 3.00 & 1.13 & 7.10 & 1.21 & 7.70 \\
\hline maw1 & 2.41 & 1.74 & 0.31 & 0.64 & 22.5 & 0.45 & 18.80 & 0.48 & 19.80 & 0.49 & 20.50 \\
\hline $\mathrm{mcm} 4$ & 2.56 & 1.66 & 0.31 & 0.23 & 10.9 & -0.42 & -16.50 & 0.81 & 31.60 & 0.80 & 31.40 \\
\hline mdo1 & 11.08 & 7.31 & 0.34 & 1.77 & 15.4 & 0.44 & 4.00 & 1.23 & 11.10 & 1.28 & 11.60 \\
\hline medi & 19.21 & 8.47 & 0.32 & 2.18 & 10.9 & 0.78 & 4.10 & 1.34 & 7.00 & 1.42 & 7.40 \\
\hline mets & 12.21 & 6.78 & 0.23 & 1.37 & 10.7 & 0.52 & 4.30 & 0.72 & 5.90 & 0.80 & 6.60 \\
\hline mkea & 2.79 & 2.34 & 0.33 & 0.56 & 18.4 & 0.25 & 9.00 & 0.61 & 22.00 & 0.61 & 22.00 \\
\hline monp & 7.14 & 5.38 & 0.26 & 1.45 & 20.3 & -0.03 & -0.40 & 0.97 & 13.60 & 0.97 & 13.60 \\
\hline nano & 15.69 & 6.01 & 0.28 & 1.75 & 10.8 & 0.61 & 3.90 & 1.41 & 9.00 & 1.39 & 8.90 \\
\hline nlib & 17.97 & 11.40 & 0.37 & 2.17 & 11.9 & 0.31 & 1.70 & 1.25 & 6.90 & 1.30 & 7.20 \\
\hline nrc1 & 15.09 & 10.11 & 0.28 & 1.93 & 12.1 & 0.83 & 5.50 & 1.00 & 6.70 & 1.12 & 7.40 \\
\hline onsa & 13.89 & 6.86 & 0.24 & 1.55 & 10.5 & 0.79 & 5.70 & 0.78 & 5.60 & 0.90 & 6.50 \\
\hline penc & 16.24 & 7.89 & 0.28 & 1.62 & 9.6 & 0.52 & 3.20 & 0.84 & 5.20 & 0.91 & 5.60 \\
\hline pert & 17.12 & 6.55 & 0.34 & 2.24 & 12.4 & 0.97 & 5.70 & 0.99 & 5.80 & 1.15 & 6.70 \\
\hline pie1 & 8.58 & 6.07 & 0.32 & 1.29 & 14.4 & 0.33 & 3.80 & 1.09 & 12.70 & 1.13 & 13.20 \\
\hline pin1 & 9.21 & 6.23 & 0.27 & 1.70 & 18.2 & 0.14 & 1.60 & 1.47 & 16.00 & 1.47 & 15.90 \\
\hline pol2 & 9.12 & 5.57 & 0.28 & 1.81 & 16.7 & 1.71 & 18.70 & 1.93 & 21.20 & 1.89 & 20.70 \\
\hline pots & 14.90 & 7.28 & 0.27 & 1.56 & 9.8 & 0.94 & 6.30 & 0.75 & 5.10 & 0.81 & 5.50 \\
\hline quin & 10.96 & 4.17 & 0.30 & 1.75 & 16.2 & -0.14 & -1.30 & 1.18 & 10.80 & 1.19 & 10.90 \\
\hline reyk & 11.48 & 5.30 & 0.28 & 1.26 & 10.3 & 0.69 & 6.00 & 0.70 & 6.10 & 0.78 & 6.80 \\
\hline rock & 12.33 & 5.93 & 0.28 & 2.40 & 18.9 & 0.34 & 2.80 & 1.50 & 12.10 & 1.65 & 13.30 \\
\hline sant & 12.28 & 4.66 & 0.28 & 2.86 & 25.7 & -1.16 & -9.40 & 2.34 & 19.10 & 2.48 & 20.20 \\
\hline shao & 27.66 & 18.21 & 0.37 & 3.38 & 11.9 & 0.73 & 2.60 & 1.41 & 5.10 & 1.76 & 6.40 \\
\hline sio3 & 16.32 & 7.70 & 0.29 & 2.41 & 14.5 & 0.23 & 1.40 & 1.52 & 9.30 & 1.61 & 9.80 \\
\hline sni1 & 12.49 & 5.91 & 0.25 & 1.91 & 14.5 & 0.71 & 5.70 & 1.28 & 10.20 & 1.29 & 10.30 \\
\hline spk1 & 12.57 & 6.11 & 0.27 & 2.42 & 18.5 & 0.49 & 3.90 & 1.49 & 11.80 & 1.54 & 12.20 \\
\hline stjo & 14.32 & 8.76 & 0.28 & 2.05 & 13.8 & 0.59 & 4.10 & 0.84 & 5.80 & 1.07 & 7.50 \\
\hline svtl & 12.63 & 7.38 & 0.30 & 1.36 & 10.3 & 0.60 & 4.70 & 0.86 & 6.80 & 0.88 & 7.00 \\
\hline syog & 3.00 & 1.78 & 0.34 & 0.75 & 20.8 & 0.62 & 20.70 & 0.58 & 19.40 & 0.58 & 19.40 \\
\hline tabl & 5.86 & 3.79 & 0.33 & 1.22 & 21.6 & -0.24 & -4.10 & 0.69 & 11.80 & 0.71 & 12.10 \\
\hline tidb & 13.97 & 6.58 & 0.30 & 1.90 & 12.9 & 0.70 & 5.00 & 1.06 & 7.60 & 1.12 & 8.00 \\
\hline tow2 & 33.37 & 13.02 & 0.28 & 3.46 & 10.4 & -0.08 & -0.20 & 1.61 & 4.80 & 1.75 & 5.20 \\
\hline trak & 15.24 & 7.10 & 0.34 & 2.28 & 14.4 & 0.55 & 3.60 & 1.71 & 11.20 & 1.74 & 11.40 \\
\hline tskb & 24.13 & 16.59 & 0.33 & 3.09 & 12.1 & 1.34 & 5.60 & 1.26 & 5.20 & 1.33 & 5.50 \\
\hline uclp & 15.40 & 6.98 & 0.29 & 2.30 & 14.8 & 0.13 & 0.80 & 1.72 & 11.20 & 1.72 & 11.20 \\
\hline uclu & 15.60 & 6.05 & 0.30 & 2.02 & 12.6 & 0.44 & 2.90 & 0.98 & 6.30 & 1.06 & 6.80 \\
\hline usc1 & 15.82 & 7.21 & 0.39 & 2.38 & 14.6 & 0.52 & 3.30 & 1.77 & 11.20 & 1.79 & 11.30 \\
\hline usud & 13.29 & 10.31 & 0.33 & 2.06 & 15.3 & 0.22 & 1.60 & 1.10 & 8.30 & 1.13 & 8.50 \\
\hline vill & 14.74 & 5.88 & 0.31 & 1.72 & 11.1 & 0.75 & 5.10 & 1.16 & 7.90 & 1.19 & 8.10 \\
\hline vndp & 14.99 & 5.93 & 0.29 & 2.85 & 18.1 & 0.76 & 5.10 & 1.39 & 9.30 & 1.40 & 9.30 \\
\hline wes2 & 16.77 & 10.87 & 0.33 & 2.18 & 12.6 & 0.48 & 2.80 & 1.07 & 6.40 & 1.12 & 6.70 \\
\hline whc1 & 15.92 & 7.21 & 0.27 & 2.32 & 14.6 & -0.05 & -0.30 & 1.77 & 11.10 & 1.87 & 11.80 \\
\hline whit & 6.47 & 3.88 & 0.26 & 0.77 & 10.8 & 0.66 & 10.10 & 0.58 & 9.00 & 0.59 & 9.20 \\
\hline will & 9.26 & 4.64 & 0.25 & 0.98 & 9.9 & 0.68 & 7.40 & 0.83 & 9.00 & 0.87 & 9.40 \\
\hline wlsn & 7.05 & 4.50 & 0.33 & 1.49 & 21.2 & 0.01 & 0.10 & 0.90 & 12.80 & 0.97 & 13.80 \\
\hline wslr & 11.81 & 4.94 & 0.35 & 1.48 & 13.2 & -0.60 & -5.10 & 1.10 & 9.30 & 1.10 & 9.30 \\
\hline wtzr & 13.52 & 6.70 & 0.25 & 1.54 & 11.0 & 0.52 & 3.90 & 0.78 & 5.80 & 0.89 & 6.60 \\
\hline wuhn & 29.75 & 17.96 & 0.34 & 3.17 & 10.2 & 1.27 & 4.30 & 1.68 & 5.60 & 1.82 & 6.10 \\
\hline yell & 9.05 & 6.65 & 0.28 & 1.00 & 10.2 & 0.70 & 7.80 & 0.90 & 10.00 & 0.93 & 10.30 \\
\hline zimm & 12.78 & 6.23 & 0.27 & 1.81 & 13.9 & 0.27 & 2.10 & 0.91 & 7.20 & 1.04 & 8.10 \\
\hline
\end{tabular}

